\title{
D éveloppement territorial en milieu rural : quelques exemples en Région wallonne
}

\author{
Marie-Françoise Godart et Mireille Deconinck \\ Université Libre de Bruxelles ${ }^{1}$
}

La régionalisation de la Belgique a amené d'importantes réformes institutionnelles, accompagnées le plus souvent de réformes dans le mode de prise de décision. C'est ainsi que dès la fin des années 1980, la Région wallonne a entrepris de développer plusieurs outils de portée communale, destinés à favoriser la participation et la prise en main, par les communes, de leur développement (schéma de structure communal, programme communal de développement territorial, plan communal de développement de la nature, plan communal général d'égouttage,...). Ces outils étaient exclusivement destinés aux entités communales, ce qui limitait fortement les actions coordonnées. Rapidement, des outils à portée plus large, rassemblant plusieurs communes ou parties de communes, mieux adaptés à une gestion optimale des territoires, tenant compte des contraintes physiques, biologiques et humaines ont été mis sur pied. Le présent article passe en revue quelques-uns de ces outils et tente de voir dans quelle mesure ils sont applicables et complémentaires.

\section{La Région wallonne²}

Sur le plan institutionnel, la Belgique est régie par trois niveaux de pouvoirs: l'État fédéral, les Régions et les Communautés. La plupart des compétences concernant directement ou indirectement l'aménagement et la gestion sont du ressort régional. Il s'agit notamment de l'aménagement du territoire et de l'urbanisme, de l'environnement et de la conservation de la nature, des richesses naturelles, de la politique de l'eau et de l'énergie, des travaux publics, des transports, du logement, de la politique économique régionale, de la politique régionale de l'emploi, de la politique agricole régionale, des pouvoirs locaux, des bâtiments et infrastructures scolaires et sportifs, du tourisme, etc.

Sur le plan institutionnel, la Belgique est régie par trois niveaux de pouvoirs: l'État fédéral, les Régions et les Communautés. La plupart des compétences concernant directement ou indirectement l'aménagement et la gestion sont du ressort régional.

La Région wallonne couvre une superficie de $16844 \mathrm{~km}^{2}$, soit plus de la moitié de la Belgique (31,7\% du territoire wallon est occupé par des prairies, $26,3 \%$ par des cultures,
$31,4 \%$ par des forêts et $11,2 \%$ est construit : bâtiments et voies de communications).

Occupant une position relativement centrale en Europe de l'Ouest, à proximité de Bruxelles, la région wallonne ou Wallonie - constitue la partie sud de la Belgique. Le relief de la Wallonie est assez accidenté; l'altitude au nord de la région est de moins de 25 mètres et s'élève progressivement entre 50 et 200 mètres sur les plateaux de Moyenne Belgique pour atteindre vers le sud et l'est une altitude culminant à 694 mètres sur le plateau des Hautes Fagnes. Vers l'extrême sud régional, au-delà du plateau ardennais, l'altitude diminue vers la Lorraine belge qui constitue la bordure nord du bassin parisien. La diversité du relief et la variété des paysages qui en résulte s'expliquent par la grande variété du sous-sol wallon.

La Wallonie comptait au $1^{\text {er }}$ janvier 1997 une population de 3320805 habitants, répartie inégalement au sein de 262 communes. La densité moyenne, de 196 hab. $/ \mathrm{km}^{2}$, est supérieure à la moyenne européenne, mais reste inférieure à celle rencontrée dans les régions voisines (Flandre et Pays-Bas notamment). La population est assez inégalement répartie sur le territoire. L'occupation humaine s'est principalement développée le long du sillon industriel formé par les vallées de la Sambre et de la Meuse (bassins charbonniers) et au nord de celui-ci (triangle Bruxelles- 
Mons-Namur). La dispersion des lieux d'habitat est beaucoup plus grande dans le sud de la région, où l'on note seulement quelques petits pôles urbanisés. Sur le plan historique, les implantations ont été influencées par les potentialités industrielles, d'échanges et de transport.

Les réseaux de communication (routes, rail, eau) irriguant la région sont assez denses. Le réseau routier se structure principalement en un réseau constitué d'autoroutes et de voies rapides, et un réseau interurbain dont le rôle est de permettre l'accessibilité, le trafic de transit étant secondaire. À ces voies principales s'ajoutent les réseaux locaux de voies communales. La Wallonie compte également deux aéroports régionaux: un situé près de Liège, l'autre près de Charleroi. Enfin, il convient de signaler que le territoire wallon se caractérise par son ouverture par rapport aux régions et pays voisins : absence de réelles barrières physiques, prolongement de ses caractéristiques géographiques au-delà des frontières dans toutes les directions.

\section{Le milieu rural en Région wallonne}

En 1991, dans le cadre du développement rural ${ }^{3}$, le gouvernement wallon a commandé au GEVERU ${ }^{4}$ une étude portant sur les critères de ruralité. Cette étude avait pour objet «la sélection d'un certain nombre de paramètres pertinents qui caractérisent l'espace rural, une typologie des régions rurales wallonnes, ainsi qu'une mise au point de critères permettant une cartographie au $1 / 50000^{\mathrm{e}} \mathrm{de}$ ces régions $»$.

\section{L'espace rural existe en Wallonie, mais il a changé sous l'action d'événements qui ont influencé le développement des villes comme des campagnes.}

L'espace rural existe en Wallonie, mais il a changé sous l'action d'événements qui ont influencé le développement des villes comme des campagnes. Identifier ces espaces et en faire une typologie devaient permettre de définir les objectifs du développement rural en termes territoriaux et d'apporter un éclairage sur l'efficacité des politiques rurales.

L'étude commence par une revue bibliographique des définitions existantes de la ruralité. Il en ressort que cette notion est particulièrement complexe et difficile à cerner. Avant les années 1950, l'espace rural paraît facile à définir : il s'agit de l'espace agricole, opposé à la ville. Depuis cette époque, des mutations d'ordre économique, démographique, social et administratif ont abouti à une évolution des paysages ruraux menant à une extension des espaces urbanisés, une banalisation architecturale de l'habitat rural, des reconversions et éliminations de bâtiments agricoles traditionnels, le développement de voies de communication, etc. En outre, la ruralité dépend étroitement du contexte, car il est évident qu'elle ne présente pas les mêmes caractéristiques en France, où la densité de population avoisine les $104 \mathrm{hab} . / \mathrm{km}^{2}$ alors qu'en Région wallonne elle atteint $196 \mathrm{hab} . / \mathrm{km}^{2}$.

Parmi les critères les plus fréquemment utilisés pour définir la ruralité, on rencontre des critères physiques, des critères économiques et politiques et des critères socioculturels

Parmi les critères les plus fréquemment utilisés pour définir la ruralité, on rencontre des critères physiques (faible densité, mode de peuplement,...) ; des critères économiques et politiques (importance du secteur primaire, faible taux d'emploi,...) et des critères socioculturels (mode de vie, comportement solidaire,...).

Pour identifier cet espace, les critères statistiques devraient pouvoir fournir de bons outils d'analyse. Ces critères peuvent être réunis en plusieurs groupes parmi lesquels on peut citer: la démographie (densité de population, pourcentage de population comprise dans des noyaux de plus de 50 habitants, etc.); la population active (structure de la population active selon les trois secteurs d'activités); les agriculteurs (pourcentage d'agriculteurs dans la population active) ; les navettes (pourcentage d'actifs travaillant hors de leur commune de résidence); les rapports avec la ville (concentration des services ou institutions typiquement urbains); l'occupation du sol (part des superficies agricole et forestière dans la superficie communale).

Ces différents critères ont été soumis à une analyse en composantes principales (ACP), dont la première composante reflète une opposition rural - urbain, mise en évidence par le type de logement (maisons individuelles, logement occupé par le propriétaire, bâtiments agricoles, etc.) et les jardins.

Des tentatives d'affinage des résultats ont montré que l'utilisation des données statistiques ne permettait pas d'établir des distinctions claires, dépourvues de toute 
ambiguïté. Une nouvelle approche a dès lors tenté de définir les communes qui pouvaient manifestement être exclues de la ruralité, sur la base de la répartition des espaces bâti et non bâti. Au sein de l'espace non bâti, les catégories susceptibles d'entretenir des relations de type rural avec les habitants ont été retenues : espaces agricoles, vergers, bois. Ces fragments de l'espace non bâti ont été qualifiés d'espace non bâti potentiellement rural.

Après avoir effectué le calcul des proportions d'espace non bâti potentiellement rural, il apparaît que toutes les communes wallonnes possèdent au moins $27 \%$ de ce type d'espace et que celles qui en possèdent plus de $75 \%$ présentent des caractéristiques indiscutablement rurales. Après d'autres essais de classification, basés sur divers critères, il est clairement apparu que le seuil de $50 \%$ d'espace agricole et sylvicole correspond à une réalité et permet d'exclure huit communes de l'analyse (Liège, St-Nicolas, Herstal, Quaregnon, Rixensart, Seraing, Waterloo et Charleroi).

Afin de préciser les résultats des analyses théoriques, des visites de terrain ont été effectuées, qui ont confirmé l'approche théorique et ont également montré que parmi les communes ayant entre 50 et $75 \%$ d'espace non bâti potentiellement rural, l'existence effective de caractéristiques rurales était surtout liée au mode de dispersion de l'habitat et la nature de l'espace non bâti potentiellement rural.

Les données statistiques au niveau communal ont montré leurs limites pour donner une description détaillée de la ruralité des communes wallonnes. Cependant, d'autres critères, de type qualitatif cette fois, ont été utilisés : existence de gîte rural ou de gîte à la ferme, existence d'une proposition de parc naturel, d'une police rurale, d'une zone d'habitat à caractère rural,... et ont permis d'éliminer encore quelques communes.

Afin d'affiner encore l'analyse sur les communes non exclues, une analyse de transfert (par ajustement progressif, les communes sont affectées à des groupes définis sur la base de communes types) a été réalisée en prenant en compte 29 variables traitant des caractéristiques de la population, de ses revenus, de l'occupation du sol, du type de logement, etc. Quatorze groupes de communes ont pu être mis en évidence, allant de «rural agricole» à «ville à périphérie ouvrière ». Sans s'attarder sur les détails de cette classification, on peut citer quelques groupes : le groupe «rural agricole» rassemble des com- munes très typiquement agricoles, généralement loin des zones urbaines ; le groupe « rural agricole avec petit centre d'emploi tertiaire » présente une présence notable de l'agriculture, mais avec croissance des variables indiquant un centre aux fonctions plus urbaines; le groupe «rural agricole à fonction résidentielle importante » regroupe des communes au caractère agricole évident, mais également avec une fonction résidentielle marquée (taux de navettes...) ; le groupe "périphérie industrielle (villages ouvriers) » rassemble des communes présentant un pourcentage élevé de résidents employés dans le secondaire et à fonction dortoir marquée. Au total, plus de $50 \%$ des communes présentent des caractéristiques encore clairement rurales.

\section{Le programme communal de développement rural (PCDR)}

Le développement rural intégré, aussi appelé chez nous «rénovation rurale», est une politique globale qui vise à réhabiliter le monde rural et à le revitaliser dans le respect de ses caractères propres.

\section{Le développement rural intégré, aussi appelé chez nous « rénovation rurale», est une politique globale qui vise à réhabiliter le monde rural et à le revitaliser dans le respect de ses caractères propres.}

L'étude décrite au point précédent a été réalisée en lien direct avec un texte légal adopté le 6 juin 1991: le Décret relatif au développement rural qui vise à mettre en place une politique locale qui a pour règles principales: l'information, la participation et la consultation de la population. Le décret définit le développement rural comme « un ensemble coordonné d'actions de développement, d'aménagement et de réaménagement entreprises ou conduites en milieu rural par une commune dans le but de sa revitalisation et sa restauration, dans le respect de ses caractéristiques propres, et de manière à améliorer les conditions de vie de ses habitants au niveau économique, social et culturel ». Ses objectifs sont: a) améliorer sur tous les plans la qualité de vie des populations des communes rurales en mettant notamment à profit les ressources physiques et humaines du milieu local; b) contribuer à une bonne gestion communale. Les moyens mis en œuvre sont : un programme global, à long terme ; la participation des habitants ; un financement régional, accompagné d'autres ressources. La procédure mise en place doit garantir la participation et la globalité de la réflexion. 
Pour aboutir à la mise en œuvre de cette politique, diverses ressources doivent être utilisées : il s'agit des ressources humaines, des ressources institutionnelles et des ressources documentaires. L'élément humain regroupe la population, le Conseil communal, les agents de développement (agissant sur le terrain et dont les deux rôles essentiels sont de transformer les souhaits de la population en projets réalisables et de servir de relais entre les différents intervenants) et la Commission locale de développement rural. Sur le plan institutionnel, on retrouve le plus souvent un organisme chargé d'aider à l'élaboration du programme par le biais de l'animation (le plus souvent, la Fondation rurale de Wallonie), les auteurs du PCDR et la Commission Régionale d'Aménagement du Territoire. Enfin, les outils documentaires sont le programme de développement proprement dit (y compris l'énumération des données socio-économiques de la commune); la méthodologie de la participation et ses résultats; les objectifs de développement adoptés par le Conseil communal et enfin, les projets.

Concrètement, les actions prévues dans le $\mathrm{PCDR}^{5}$ visent à : promouvoir l'emploi et les activités économiques ; protéger et mettre en valeur le cadre de vie; créer et améliorer les services à la population; promouvoir l'habitat; créer et aménager des espaces publics. Cette énumération n'est pas exhaustive, mais recouvre les principaux champs d'actions déclinés dans les PCDR existants.

L'élaboration de ces programmes repose, on l'a vu plus haut, sur la participation de la population. Concrètement, cela implique la mise en place de groupes de travail composés de représentants de la population. Ces groupes de travail sont accompagnés par un organisme actif dans la ruralité. L'organisme accompagnateur doit informer la population, constituer un dossier de base et consulter la population sur la base de ce dossier. Les groupes de travail se spécialisent généralement dans certains domaines précis (économie, agriculture, environnement, patrimoine, ...) et élaborent des projets qui seront ensuite traduits en fiches par un spécialiste. Afin de coordonner l'élaboration du programme, une Commission Locale de Développement Rural réunit entre 10 et 30 personnes, dont le quart peut être désigné au sein du Conseil communal, les autres membres devant être représentatifs des milieux politique, économique, socioprofessionnel et culturel de la commune, des différents hameaux et villages et des différentes classes d'âge, ainsi que des groupes de travail. La Commission est présidée par le Bourgmestre.
Lorsque le programme est établi, il doit être approuvé par la commune. Il est ensuite examiné par la Commission régionale d'aménagement du territoire (CRAT) qui remet un avis sur sa qualité, son opportunité, et enfin, le programme est transmis à la Région wallonne (autorités et administration). Lorsqu'il est approuvé par ces diverses instances, des conventions sont établies en vue de l'exécution des travaux. Il convient également de signaler que l'élaboration de ce programme bénéficie de subsides de la Région wallonne, désireuse d'aider les communes à l'élaborer et le mettre en oeuvre.

Cet outil de développement, spécifique aux zones rurales, a été mis en place dans de nombreuses communes. C'est ainsi qu'en mars 2000, 106 communes avaient décidé de se lancer dans la réalisation d'un tel programme et 62 l'avaient bouclé. Cet engouement a posé d'importants problèmes à la Région wallonne sur le plan budgétaire.

\section{Le Parc naturel ${ }^{6}$}

La Loi du 12 juillet 1973, dite « de la conservation de la nature», définissait divers types de territoires. Les parcs naturels sont définis comme des «territoires soumis à des mesures ayant pour but de conserver le caractère, la diversité et les valeurs scientifiques de l'environnement, la flore et la faune indigènes, ainsi que la pureté de l'air et des eaux et d'assurer la conservation de la qualité des sols ». Selon que le parc est créé sur l'initiative de l'État ou d'un autre pouvoir public, il sera appelé parc naturel national ou régional. La hiérarchie prévue offre plus de sécurité au parc national qu'au parc régional.

Après l'adoption de cette loi, plusieurs tentatives de création de parcs furent lancées. Une partie d'entre elles concernait des territoires pour lesquels une réflexion avait déjà été menée, alors que pour d'autres, c'est la piste ouverte par la loi qui a incité à la mise en place d'un processus de réflexion, prolongé par des études plus ou moins poussées. Malgré des actions parfois bien avancées, le seul projet qui arriva à une concrétisation officielle, dans le cadre de la Loi de 1973, est le Parc Naturel des Hautes Fagnes-Eifel.

Le 16 juillet 1985, un décret de l'Exécutif régional wallon relatif aux parcs naturels était adopté. Ce décret apporte une nouvelle dimension à la notion de parc naturel puisqu'il intègre l'environnement à la vie rurale, axé sur le développement harmonieux entre l'économie locale et la nature. Dans la version de 1973, il s'agissait plus spécifiquement de protection de la nature au sens large. 
Le parc naturel est défini comme suit: « un parc naturel est un territoire rural, d'un haut intérêt biologique et géographique, soumis conformément au présent décret à des mesures destinées à en protéger le milieu, en harmonie avec les aspirations de la population et le développement économique et social du territoire concerné ». Il est encore précisé que tout parc naturel couvre une superficie minimum de 5000 ha d'un seul tenant (même s'il est traversé par des lignes de chemin de fer, des autoroutes,...).

Cette nouvelle conception du parc naturel a entraîné une réorientation de certains projets, étudiés dans le cadre de la loi de 1973. Elle a aussi entraîné la conception de nouveaux projets, concernant des zones plus propices à cette nouvelle conception. C'est le pouvoir organisateur qui décide de la création du parc naturel, à condition que la majorité des communes ait émis un avis favorable. Un plan de gestion doit être établi. Il indique notamment les objectifs poursuivis en ce qui concerne la conservation de la nature, la protection de l'environnement, l'aménagement du territoire et le développement rural et économique; mais aussi les modifications éventuelles à apporter aux plans d'aménagement en rapport avec la création du parc naturel. Il indique aussi les différents moyens à utiliser pour une gestion qui atteigne les objectifs du parc.

Le parc naturel est à la fois un outil de gestion et un moyen de contrôler la pression des différentes activités humaines sur le sol.

Le parc naturel est à la fois un outil de gestion et un moyen de contrôler la pression des différentes activités humaines sur le sol. Son importance est d'autant plus grande qu'il peut concerner des étendues non négligeables de territoire (plusieurs milliers d'hectares). Or, si la protection de la nature représente un élément majeur dans le parc naturel, l'essentiel pour les habitants est de maintenir un cadre de vie agréable et viable.

Cependant, ces deux points représentent un équilibre difficile à réaliser puisqu'il faut assez de sites pour assurer le maintien de l'intérêt naturel du parc, mais pas trop pour ne pas nuire aux autres activités. De même, l'attrait de ces sites naturels ne doit pas les étouffer par une pression touristique exagérée. Sous cet angle, l'importance de la gestion apparaît pleinement, tant pour les zones naturelles que pour l'ensemble du parc.

Depuis l'adoption du décret de 1985, neuf parcs naturels régionaux ont été reconnus : celui des Hautes Fagnes-Eifel
(1978), celui des vallées de la Burdinale et de la Mehaigne (1990), celui de la vallée de l'Attert (1994), celui des Plaines de l'Escaut (1996), celui du Pays des Collines (1997), celui de Viroin-Hermeton (1998), celui des HautsPays (2000), celui de la Forêt d'Anlier (2001) et celui des Deux Ourthe (2001).

À noter que les modalités de financement des parcs par la Région n'ont jamais été arrêtées, ce qui induit des difficultés non négligeables pour la viabilité des parcs, la pérennité des moyens financiers n'étant pas assurée.

Un bilan sur le fonctionnement des parcs naturels a été effectué récemment et devrait amener des modifications concrètes du décret, destinées à le rendre plus opérationnel et, par là, rendre la notion de parc naturel plus attractive pour les territoires présentant les qualités nécessaires sur le plan de l'environnement. Près de $2000 \mathrm{~km}^{2}$ sont couverts par les parcs naturels existants et une quarantaine de communes sont concernées en tout ou en partie.

\section{Q uelques autres outils disponibles pour les communes rurales}

\section{a) le schéma de structure communal ${ }^{7}$}

Contrairement aux autres outils déjà évoqués, le schéma de structure communal est un outil mis à la disposition de toutes les communes, quelles que soient leurs caractéristiques (urbaine, rurale, industrielle, à caractère seminaturel,...). L'existence du schéma de structure communal est liée au décret sur la décentralisation et la participation promulgué en avril 1989. Dans ce document, le schéma de structure est défini comme «le document d'orientation, de gestion et de programmation du développement de l'ensemble du territoire communal». Ce schéma est également un outil indispensable pour permettre à la commune d'accéder à un régime de plus grande autonomie en ce qui concerne la gestion de son territoire (particulièrement du point de vue de l'aménagement du territoire). Il permet enfin d'engager les habitants dans un processus participatif, leur donnant l'occasion, non seulement de s'exprimer, mais aussi de contribuer à guider l'évolution de leur cadre de vie.

Dans la pratique, le schéma de structure répond de manière diversement satisfaisante à ces objectifs et sert essentiellement d'instrument pour la délivrance des permis d'urbanisme et de lotir ${ }^{8}$. Comme la plupart des outils mis à disposition des communes par la Région, celui-ci est également subsidié en tout ou en partie par la Région. 


\section{b) Le Plan Communal de Développement de la Nature (PCDN) ${ }^{9}$}

Le PCDN peut être vu comme une démarche stratégique de la Région vis-à-vis des communes. La volonté est d'étendre cette opération aux 262 communes wallonnes, établissant une stratégie globale et cohérente pour la biodiversité proposée à l'ensemble de la Région ${ }^{10}$. Les objectifs de ces plans sont de: "préserver ou améliorer le patrimoine naturel et paysager d'un territoire, tout en respectant et favorisant le développement économique et social des habitants. Il s'agit de promouvoir le développement des valeurs naturelles et paysagères et de maintenir ou reconstituer un réseau écologique au niveau communal, en faisant participer tous les acteurs locaux concernés. Ce partenariat vise à sensibiliser chaque acteur au caractère commun du patrimoine naturel communal et à sa prise en charge au profit des générations futures; il doit renforcer l'identité de chaque acteur dans la réalisation d'un projet commun.»

On le voit, ce plan est sectoriel, mais le fait qu'il s'agisse d'un plan introduit l'idée de la planification, de la programmation en matière de protection du patrimoine naturel, où le partenariat joue un rôle essentiel visant deux objectifs : la sensibilisation du plus grand nombre et la responsabilisation de chacun à travers la réalisation d'un projet. Il s'agit aussi, et il importe de le souligner, d'assurer la protection du patrimoine naturel et paysager dans le cadre du développement économique et social des habitants ${ }^{11}$.

Le PCDN se déroule en six étapes :

- établissement d'un partenariat aussi large que possible entre les «acteurs-utilisateurs » de l'espace rural communal (CCAT, entreprises, écoles, associations de protection de la nature,...);

- réalisation d'un inventaire du patrimoine naturel et paysager de la commune; élaboration d'une carte du réseau écologique, permettant la mise en évidence des points forts et des points faibles de la commune (l'inventaire est fait par un expert d'un bureau d'étude);

- organisation d'un partenariat en groupes de travail (en parallèle avec la réalisation de l'inventaire) pour aboutir à des «fiches-action»;

- intégration des «fiches-action» et de la cartographie du réseau (attention: respecter les principes du PCDN, ne pas être trop ponctuel);
- élaboration d'un plan de réalisation (sur 4 ans): actions concrètes à court, moyen et long terme pour maintenir, protéger, améliorer et restaurer la qualité du milieu naturel et paysager communal ;

- signature, par tous les partenaires, d'un «contrat», dans lequel chacun s'engage à concrétiser le PCDN.

Dans ce cadre, des subsides peuvent être octroyés aux communes, mais celles-ci doivent aussi prévoir un budget alloué à l'étude et/ou à la dynamique de réalisation. Outre ces actions, une large part de la démarche doit être réservée à l'information, à la sensibilisation et à l'éducation de la population. Un budget communal sera affecté à la démarche pour couvrir les frais de fonctionnement du partenariat et de la communication vers le grand public.

Le PCDN n'a aucune force réglementaire. Il s'agit d'un plan élaboré volontairement par un groupe de personnes, dont le représentant communal, pour tracer les lignes directrices à suivre concernant la gestion communale de la biodiversité dans les cinq années à venir. Il s'appuie sur une implication forte de la commune et un partenariat local actif.

Si le PCDN est un outil de fédération au sein d'une commune pour restaurer ou préserver la biodiversité, d'autres outils, plus ponctuels ou au contraire plus larges, ont également été mis sur pied par la Région wallonne. C'est le cas d'une série d'actions qui donnent droit à des subsides : diverses mesures d'aide à la gestion des forêts privées ; les mesures agri-environnementales ; les plantations de haies; l'encadrement des activités de loisirs par des mesures d'amélioration des camping-caravaning ; des mesures visant à promouvoir le tourisme rural (gîtes ruraux, à la ferme, etc.).

\section{c) Le contrat de rivière}

Tel que défini dans la Circulaire ministérielle relative aux conditions d'acceptabilité et aux modalités d'élaboration des contrats de rivière en Région wallonne du 18 mars $1993^{12}$, le contrat de rivière est « un protocole d'accord entre l'ensemble des acteurs publics et privés sur des objectifs visant à concilier les multiples fonctions et usages des cours d'eau, de leurs abords et des ressources en eau du bassin ». Ce protocole peut être institué sous forme de charte.

Dans cette démarche, les acteurs locaux doivent donc étudier les actions qu'ils peuvent eux-mêmes mettre en 
œuvre pour atteindre les objectifs qu'ils se fixent dans les limites géographiques visées par le contrat et dont l'axe est la rivière. Divers aspects sont à prendre en compte : restaurer, protéger et valoriser les ressources en eau du bassin en intégrant harmonieusement l'ensemble des caractéristiques propres à la rivière; sensibilisation, information et participation de l'ensemble des acteurs résidant dans la zone (développement d'une dynamique durable).

La circulaire fixe les modalités d'élaboration du contrat:

- initialisation du projet: cette étape, menée par les promoteurs du projet (communes, institutions ou associations privées) comprend la constitution d'un dossier préparatoire et la rédaction d'une convention d'étude;

- approbation du projet de convention d'étude (et désignation d'un auteur de projet) par le ministre compétent et réservation de subsides;

- exécution de la convention, avec mise en place du Comité de rivière (représentants des usagers et gestionnaires du bassin). Cette exécution implique notamment l'inventaire des problèmes, l'identification de solutions et la définition d'un programme d'actions local et cohérent;

- signature du Contrat de rivière (soit d'un programme d'action, qui doit faire l'objet d'une large publicité);

- mise en place d'un comité de suivi et exécution des engagements par les signataires.

Il importe de préciser que le financement de la convention d'étude et du comité de suivi est assuré par la Région, les provinces, les communes et tout autre partenaire désireux de soutenir financièrement le projet.

L'expérience du contrat de rivière de la Haute Meuse montre que les domaines d'action d'un contrat de rivière sont divers et débordent largement de la dimension «eau ». De plus, parmi les objectifs de tels contrats, assurer la qualité de l'eau, sa fourniture en quantité suffisante et à un prix abordable sont de puissants incitants économiques pour garantir une bonne implication de la commune.

En 2002, treize contrats de rivière, couvrant une part non négligeable du territoire wallon (soit 110 communes et $6486 \mathrm{~km}^{2}$ ) avaient vu le jour (Dendre, Dyle, Haute-Meuse, Hoegne et Wayai, Honnelles, Munos, Sambre, Semois,
Ton, Trouille, Vesdre, Wiltz). À noter que cette opération de partenariat peut concerner tant de grandes rivières (Semois, Sambre) que de petits affluents (Ton).

\section{Conclusion}

Les quelques outils présentés ci-dessus montrent la variété des moyens d'action mis à la disposition des collectivités territoriales pour agir sur leur développement. On se rend bien compte de la complexité devant laquelle se trouve confrontée une commune voyant à sa disposition tant d'outils dont elle ne comprend pas toujours la portée et dont elle ne voit pas toujours la spécificité. Or, un outil mal compris sera un outil mal utilisé. De plus, nombre de communes, et plus particulièrement de communes rurales, ne disposent pas d'un personnel suffisamment qualifié, pour appréhender les particularités, les inconvénients et les avantages de tous ces outils. Si la Région met le plus souvent des moyens financiers à la disposition des communes pour les aider à financer l'établissement des différents outils signalés, elle leur donne beaucoup plus rarement les moyens humains nécessaires à la bonne gestion et l'application de ces outils.

Une exception notable doit être signalée: depuis plus de 10 ans, les communes qui en font la demande peuvent bénéficier d'aide pour engager des «conseillers en environnement », à la condition que ceux-ci aient les titres requis. Cependant, cette aide est limitée dans le temps et de nombreuses communes hésitent à pérenniser la fonction lorsqu'elles doivent prendre en charge la totalité du traitement de ces conseillers. Le gouvernement, conscient des problèmes posés par la multiplication des outils, mais surtout par l'augmentation des responsabilités dévolues aux communes, prévoit de mettre sur pied une formation adaptée pour le personnel communal et financer des conseillers en environnement et aménagement du territoire.

Il ne faut cependant pas noircir le tableau : certaines communes, plus dynamiques et volontaristes que d'autres, ont pleinement profité des différents outils et ont pu jouer de leur complémentarité pour valoriser leur territoire au maximum. Ces exemples restent malheureusement l'exception et il faut espérer que les nouveaux conseillers, dont l'existence même et le rôle restent à définir, pourront œuvrer dans un proche avenir à aider les communes rurales à valoriser leur territoire au moyen des outils mis à leur disposition. Ceci dit, les moyens humains ne suffisent pas, une meilleure complémentarité des outils devrait également être assurée. Des pistes de rationalisa- 
tions ont été évoquées (notamment au travers des travaux de la CPDT), mais elles ne prenaient pas en compte les outils supracommunaux, pourtant indispensables pour appréhender les problèmes d'environnement à une échelle pertinente.

\section{II existe un grand intérêt pour le développement territorial en milieu rural et sa nécessaire évolution, mais ce développement et ses applications sont actuellement en pleine mutation.}

De ce qui précède, on voit qu'il existe un grand intérêt pour le développement territorial en milieu rural et sa nécessaire évolution, mais que ce développement et ses applications sont actuellement en pleine mutation, notamment sous l'impulsion des règlements européens. Parmi ceux-ci on peut citer la directive relative à l'évaluation environnementale des plans et programmes (directive 2001/ 42/CE du 27 juin 2001), ainsi que l'obligation de réaliser des programmes de développement des zones rurales.

\section{Notes et références}

1 Marie-Françoise Godart est Dr. Sc. botaniques, professeure à l'IGEAT (Institut de gestion de l'environnement et d'aménagement du territoire) à l'Université Libre de Bruxelles (ULB). Mireille Deconinck est Dr. Sc. géographiques, professeure à l'Université Libre de Bruxelles (ULB).

2 Schéma de développement de l'espace régional (SDER), mai 1999.
3 Voir «Le programme de développement rural» à la section suivante.

4 GEVERU (Groupe d'étude pour la valorisation de l'espace rural et urbain) (L. Bronchart, I. Denonville et P. Marissal) (1991). «Critères de ruralité », Rapport final, Étude financée par le ministre de la Région wallonne ayant la rénovation rurale dans ses attributions. Le GEVERU a été incorporé en 1993 à l'IGEAT (Institut de gestion de l'environnement et d'aménagement du territoire).

5 Arrêté de l'Exécutif régional wallon portant exécution du décret du 6 juin 1996 relatif au développement rural.

6 Sites internet: http://environnement.wallonie.be/cgi/dgrne ; http://mrw.wallonie.be/dgrne/aerw/pnat/tdmpnat.htm\#tete

7 Décret de décentralisation et de participation modifiant le CWATUP du 27 avril 1989.

8 Arrêté de l'Exécutif régional wallon du 5 avril 1990 déterminant le contenu du dossier d'un schéma de structure communal en matière d'aménagement du territoire et d'urbanisme.

9 Décret du 22 janvier 1998 qui inscrit une section 5 dans le décret wallon du 21 avril 1994 relatif à la planification en matière d'environnement dans le cadre du développement durable.

10 Plan d'Environnement pour un Développement Durable (PEDD) - 1995.

11 CPDT (1999). Thème 7.5 : Rationalisation des outils de développement local, rapport final, (GUIDE - ULB ; CREAT - UCL; LEPUR - ULG), étude financée par le ministère de la Région wallonne; CPDT (2000). Thème 7.5 : Rationalisation des outils de développement local, résumé du rapport final de la subvention 1999, (GUIDE - ULB ; CREAT - UCL ; LEPUR - ULG), étude financée par le ministère de la Région wallonne.

12 Circulaire ministérielle du 18 mars 1993 relative aux conditions d'acceptabilité et aux modalités d'élaboration des contrats de rivière en Région wallonne; circulaire du 18 juin 1996, modifiée par celle du 3 juin 1997. 